\title{
Confirming an integrated pathology of diabetes and its complications by molecular biomarker-target network analysis
}

\author{
ZIDE ZHAO $^{1 *}$, YINGYING ZHANG ${ }^{2,3^{*}}$, FENGCHUN GAI $^{4}$ and YING WANG $^{1}$ \\ ${ }^{1}$ Department of Neuro-Ophthalmology, Eye Hospital, China Academy of Chinese Medical Sciences, Beijing 100040; \\ ${ }^{2}$ Laboratory of Pharmacology, Institute of Basic Research in Clinical Medicine, \\ China Academy of Chinese Medical Sciences, Beijing 100700; ${ }^{3}$ Ministry of Scientific Research, \\ Shaanxi Buchang Pharmaceutical Co. Ltd, Xi'an, Shaanxi 712000; ${ }^{4}$ Department of Infection Control, \\ The Affiliated Hospital to Changchun University of Chinese Medicine, Changchun, Jilin 130021, P.R. China
}

Received August 29, 2015; Accepted May 27, 2016

DOI: $10.3892 / \mathrm{mmr} .2016 .5478$

\begin{abstract}
Despite ongoing research into diabetes and its complications, the underlying molecular associations remain to be elucidated. The systematic identification of molecular interactions in associated diseases may be approached using a network analysis strategy. The biomarker-target interrelated molecules associated with diabetes and its complications were identified via the Comparative Toxicogenomics Database (CTD); the Search Tool for Recurring Instances of Neighboring Genes was utilized for network construction. Functional enrichment analysis was performed with Database for Annotation, Visualization and Integrated Discovery software to investigate connections between diabetes and its complications. A total of 142 (including 122 biomarkers, 10 therapeutic targets and 10 overlapping molecules) biomarker-target interrelated molecules associated with diabetes and its complications were identified via the CTD database, and analysis of the network yielded 1,087 biological processes and fifteen Kyoto Encyclopedia of Genes and Genomes pathways with significant P-values. Various critical aspects of the networks were examined in the present study: a) Intermolecular horizontal and vertical combinations in biomarkers and therapeutic targets associated with diabetes and its complicationb) network topology properties associated with molecular pathological responsec) contribution of key molecules to integrated regulation; and d) crosstalk between multiple pathways. Based on a multi-dimensional analysis, it was concluded that the integrated molecular pathological development of diabetes and
\end{abstract}

Correspondence to: Professor Ying Wang, Department of Neuro-Ophthalmology, Eye Hospital, China Academy of Chinese Medical Sciences, 33 Lugu Road, Beijing 100040, P.R. China

E-mail: tcmwangying@126.com

*Contributed equally

Key words: diabetes, complications, integrated analysis, network, biomarker-target its complications does not proceed randomly, which suggests a requirement for integrated, multi-target intervention.

\section{Introduction}

Diabetes is a group of metabolic diseases rapidly increasing as a result of an aging population, urbanization and associated lifestyle changes. In 2010, >250 million people worldwide had type 2 diabetes mellitus (1), and this number continues to rise. Considerable improvements have occurred in the detection of this disease and in effective interventions in highrisk populations (2). Despite improvements in blood glucose control and increased public awareness of type 2 diabetes mellitus over the past decade, human obesity, and therefore diabetes, remains a challenge. As a chronic metabolic disease, type 2 diabetes mellitus and its complications, including diabetic nephropathy (prevalence, 5 to $20 \%$ ), neuropathy (prevalence, 8 to $68 \%$ ), angiopathy (stroke prevalence, 4 to $12 \%$ ), cardiomyopathy (prevalence, 5 to $36 \%$ ) and retinopathy (prevalence, 11 to $65 \%$ ) (3), are a major global healthcare concern. The induction of endothelial dysfunction (4), oxidative stress (5), immunity and inflammation $(6,7)$, and mitochondrial damage $(8,9)$ by hyperglycemia is an important aspect of the molecular pathology of diabetes and its complications. The origin of the diabetes epidemic lies in the interaction of an extremely complex combination of genetic and epigenetic predispositions, with an equally complex combination of societal factors that determine behavior and environmental risk (2). In addition, the complications of diabetes have a greater negative impact on patient quality of life and the economy compared with diabetes itself (10). Thus, the systematic elucidation of the mechanisms underlying diabetic complications is critical for improved control of this disorder.

The pathological mechanisms underlying diabetes and its complications likely reflect complex interactions between genetic and environmental factors, as well as the balance between glycemic indices and insulin (INS) secretion. The continued genome-wide identification of associated risk loci and susceptibility genes for diabetes and its complications has explained a substantial proportion of disease risk, thus serving as a proof of principle for genome-wide approaches 
for elucidation of complex genetic traits (11-16). Additional examination of underlying epigenetic mechanisms using recently developed next-generation sequencing technologies has provided novel insights into the pathology of diabetes and its complications and may facilitate the discovery of novel therapeutic targets (17). The integrated management of biomarkers and therapeutic targets may enable the early diagnosis, prevention and treatment of this complex metabolic disease. The aim of the present study was to simplify and confirm the molecular associations between diabetes and its complications based on a network analysis strategy, in order to facilitate the development of novel therapeutic agents and improve disease management.

\section{Materials and methods}

Network construction. Known biomarkers and therapeutic targets of diabetes and its complications (genes with a curated association or an inferred association via a curated chemical interaction) were retrieved from the Comparative Toxicogenomics Database (CTD; ctdbase.org/). This database was used to investigate associations between diseases and genes/proteins. Certain genes are labeled as biomarkers or targetdisease-associated biomarker and target genes were identified following screening for duplicate genes/proteins. The molecular datasets were uploaded to the Search Tool for Recurring Instances of Neighboring Genes (STRING) database version 9.05 (string-db.org/) to characterize the associations between these molecules and generate molecular interaction networks. Human data were used in the present study to analyze the pathological and pharmacological correlations between diabetes and its complications. The confidence of the linkages between molecular nodes was determined based on the combined assessment of various data sources, including neighborhood, gene fusion, co-occurrence, co-expression, experiment, database, text mining and homology (18).

Analysis of network topology. Cytoscape software version 2.8.3, an interactive platform compatible with numerous network analysis software packages, was used for network visualization (cytoscape.org/). The use of Cytoscape for the biomarker-target-associated micro-network enabled the simultaneous identification of multiple essential parameters of the network, including size, average degree and node degree distribution. Centiscape software version 1.21 (cbmc. it/ scardonig/centiscape/centiscape.php), a Cytoscape plug-in, was used to compute specific node centrality to efficiently describe network topology.

Functional annotation of molecular clusters. The Database for Annotation, Visualization and Integrated Discovery (DAVID; david.abcc.ncifcrf.gov/) was used to obtain comprehensive explanations of molecular function and large-scale heterogeneous annotation content, including gene ontology (GO) terms, protein domains and signaling pathways, based on gene classification. In the present study, target molecule clusters were annotated by DAVID software version 6.7 (parameters, count $=2$, expression analysis systematic explorer $=0.01$ and species=Homo sapiens). Biological processes corresponding to the molecular clusters were identified using GO annotation and the P-values of the biological processes obtained from $\mathrm{GO}$ enrichment were ranked.

Statistical analysis. The threshold of EASE Score, a modified Fisher Exact P-value, was used for gene-enrichment analysis. $\mathrm{P}$-value ranged from 0 to 1 . A Fisher Exact P-value of 0 represents perfect enrichment. $\mathrm{P}<0.05$ was considered to indicate a statistically significant difference in the annotation categories (default is 0.1). When members of two independent groups fall into one of two mutually exclusive categories, Fisher Exact test is used to determine whether the proportions of those falling into each category differ by group. In DAVID annotation system, Fisher Exact test was performed to measure the gene-enrichment in annotation terms.

\section{Results}

Biomarker-target molecular networks. A total of 142 biomarker-target interrelated molecules associated with diabetes and its complications were identified as network nodes using the CTD database, including 122 biomarkers, 10 therapeutic targets and 10 molecules overlapping biomarkers and therapeutic targets. Fig. 1 presents the biomarker-target network, which indicates scarce linkages (2 lines) between target molecules (black nodes) and abundant linkages (135 lines) between targets and biomarkers (gray nodes). The overlapping components (white nodes) between biomarkers and targets are a striking indicator of the closeness of the internal associations. Specifically, assigning a novel role (biomarker) to a target may increase the connectivity of this micro-network (white nodes). Importantly, almost all the nodes shared between therapeutic targets and biomarkers (white nodes) doubled as overlapping nodes (colorful subnetwork presented in Fig. 2) between diabetes and its complications. This multidimensional molecular cluster contributes to a highly component-dependent network skeleton that may systematically promote the co-expression of multiple complication phenotypes, representing an integrated pathological trigger.

By contrast, a different visualization layout indicates that the groups of molecules associated with each diabetic complication are relatively independent (Fig. 2). Nodes were divided into eight categories: Diabetes mellitus (red nodes), diabetic angiopathies (yellow nodes), diabetic cardiomyopathies (light purple), diabetic nephropathies (blue nodes), diabetic neuropathies (dark purple), diabetic retinopathy (green nodes), microvascular complications of diabetes (pink nodes) and mixed (multicolored), which represented 57.04\% (81/142), $2.11 \%$ (3/142), $2.82 \%$ (4/142), $9.15 \%$ (13/142), 3.52\% (5/142), $2.82 \%$ (4/142), $2.11 \%(3 / 142)$ and $20.42 \%(29 / 142)$ of the total number of nodes, respectively. The 1,274 lines that corresponded to quantitative associations between molecules were investigated in more detail using the STRING database. Although the interior is sparse, 6 complication-associated molecular clusters exhibited high external connectivity, for example 34, 36, 91, 49, 85 and 49 total connections for the molecular clusters for diabetic angiopathies, diabetic cardiomyopathies, diabetic nephropathies, diabetic neuropathies, diabetic retinopathy and microvascular complications of diabetes, respectively. 


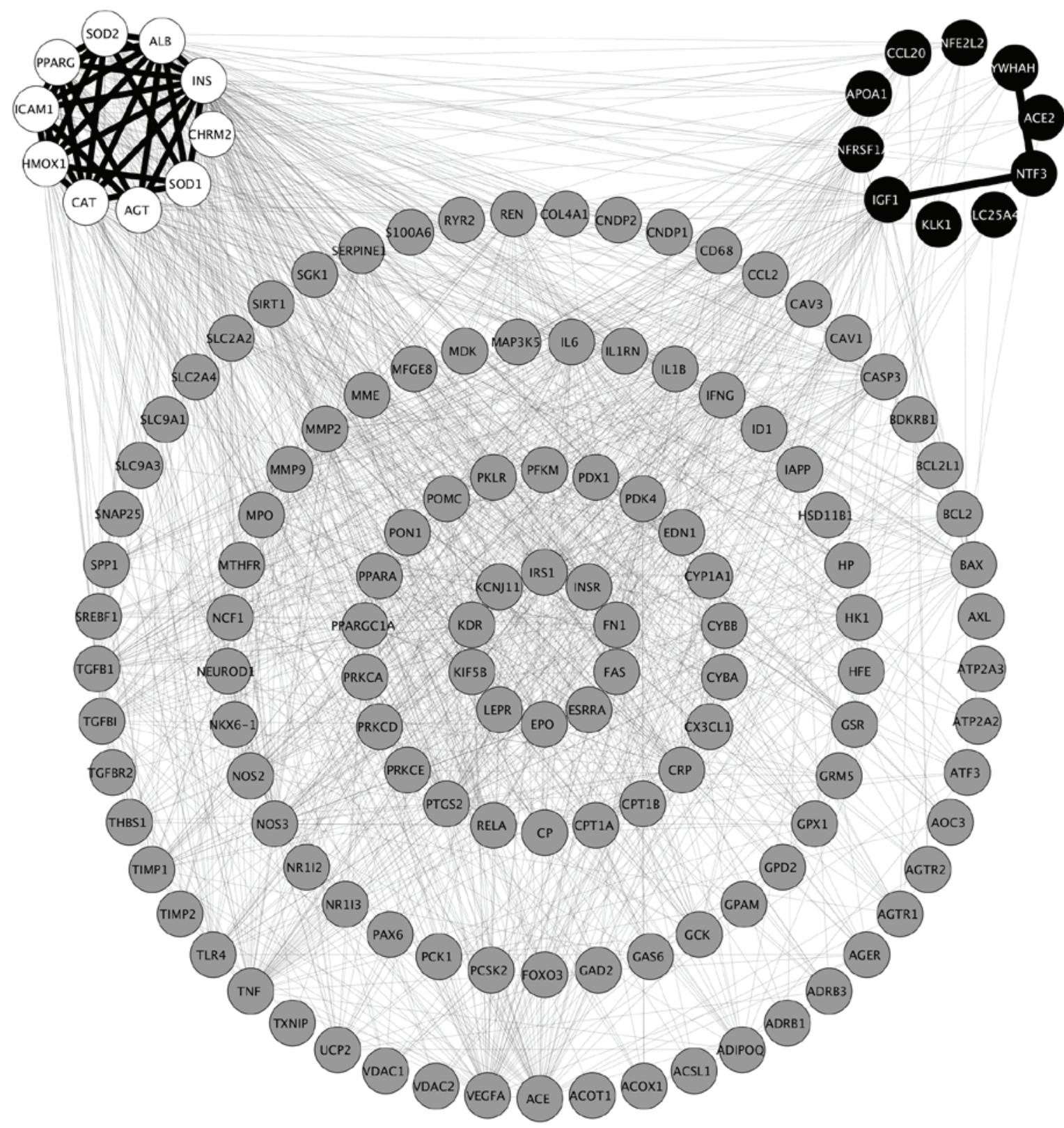

Figure 1. Biomarker-target-associated molecular networks. A total of 142 biomarker-target-interrelated molecular nodes were identified to be associated with diabetes and its complications, including 122 biomarkers (gray nodes), 10 therapeutic targets (black nodes) and 10 molecules overlapping biomarkers and therapeutic targets (white nodes). Lines between pairs of nodes represent interactions between two molecules.

Network topology analysis. In the absence of isolated nodes, the reconstructed molecular network provides a background of global pathology and drug intervention for diabetes and its complications, network analysis by Centiscape 1.21 revealed a high clustering coefficient of 0.504 [compared with a regular network and small-world network proposed based on high clustering coefficients of 0.5 and 0.453 , respectively, as defined by Watts and Strogatz (19)]. This suggests a network with markedly associated cascade-like pathways that provides the necessary conditions for combined diagnosis and treatment. Analysis of the network reveals a network diameter of 5, a network centralization of 0.526 , a characteristic path length of 2.236 and a network density of 0.127 . Taken together, these results suggest that protein-protein interactions, signal transduction, metabolic pathways or transcriptional regulation may improve the efficiency of a network response based on pathology or pharmacology. Furthermore, the average number of neighbors was 17.944 , indicating that nodes with a node degree $\geq 17.944$ were considered hubs (the top eight hubs are listed in Table I). A total of fifty-two hubs were significantly enlarged in this network to describe the basic framework (Fig. 3A) of the region of concentrated intermolecular communication.

The existence of hub nodes is an important feature of network heterogeneity. A total of fifty-two nodes were above the average degree of network nodes (Fig. 3A). In addition, $43.4 \%$ of the hubs were part of the aforementioned multidimensional molecular clusters. The top ten hubs accounted for up to $80 \%$ of these clusters. To reduce the risk of artifacts due to artificial networks, hubs with high ranks were investigated. Hyperglycemia induces the development of complications through altered gene expression and/or protein function, which contributes to cellular dysfunction and damage. INS molecules 


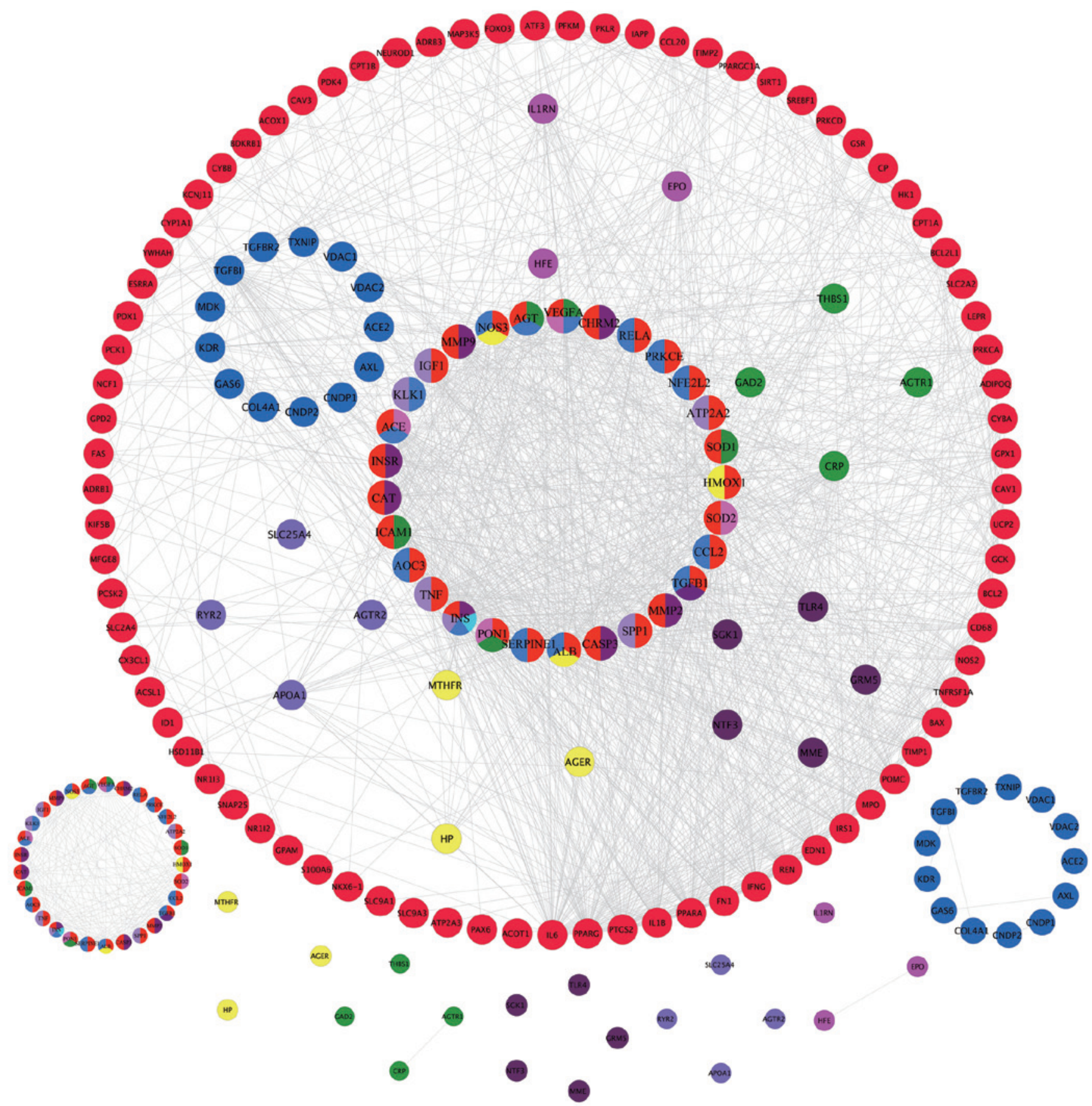

Figure 2. Molecular networks associated with diabetes and its complications. A total of 142 biomarker-target-interrelated molecular nodes were divided into eight subgroups: Diabetes mellitus (red nodes), diabetic angiopathies (yellow nodes), diabetic cardiomyopathies (light purple), diabetic nephropathies (blue nodes), diabetic neuropathies (dark purple), diabetic retinopathy (green nodes), microvascular complications of diabetes (pink nodes) and a mixed (multicolored). Lines represent interactions between any pair of intra- or intergroup nodes.

may be central. As a shared node between diabetes and four complications: Cardiomyopathies, ketoacidosis, nephropathies and neuropathies, INS exhibited connections with other high-weight hubs, including albumin, tumor necrosis factor (TNF), vascular endothelial growth factor (VEGF) A, peroxisome proliferator-activated receptor $\gamma$, superoxide dismutase 2, mitochondrial (SOD2), transforming growth factor $\beta 1$ (TGF $\beta 1$ ) and angiotensin I converting enzyme. Thus, INS forms an interactive network based on the STRING database (Fig. 3B). Molecular interactions revealed horizontal or vertical information flow and primarily included binding, activation and the inhibition or induction of gene expression. Furthermore, these hub nodes completely covered the targets and biomarkers of diabetes and its complications. The statistics on the associations between these hubs, diabetes and its complications are listed in Table I.

Functional enrichment analysis. Network analysis using DAVID software yielded 1,087 biological processes, and fifteen pathways from Kyoto Encyclopedia of Genes and Genomes (KEGG), with significant P-values. The top 30 significant biological processes are listed in Table II, including homeostatic 
Table I. Literature and statistics-based association verification between top hubs and diabetes and its complications.

A, Insulin hub gene

\begin{tabular}{lc}
\hline Condition & Reference counting $^{\mathrm{a}}$ \\
\hline Diabetes mellitus & 331 \\
Diabetic angiopathies & 9 \\
Diabetic cardiomyopathies & 25 \\
Diabetic nephropathies & 107 \\
Diabetic neuropathies & 49 \\
Diabetic retinopathy & 14 \\
Microvascular complications of diabetes & 0 \\
\hline
\end{tabular}

$\mathrm{B}$, Albumin hub gene

\begin{tabular}{lc}
\hline Condition & Reference counting $^{\mathrm{a}}$ \\
\hline Diabetes mellitus & 326 \\
Diabetic angiopathies & 8 \\
Diabetic cardiomyopathies & 26 \\
Diabetic nephropathies & 120 \\
Diabetic neuropathies & 49 \\
Diabetic retinopathy & 16 \\
Microvascular complications of diabetes & 0 \\
\hline
\end{tabular}

C, Vascular endothelial growth factor A hub gene

\begin{tabular}{lc}
\hline Condition & Reference counting \\
\hline Diabetes mellitus & 336 \\
Diabetic angiopathies & 12 \\
Diabetic cardiomyopathies & 27 \\
Diabetic nephropathies & 126 \\
Diabetic neuropathies & 51 \\
Diabetic retinopathy & 27 \\
Microvascular complications of diabetes & 1 \\
\hline
\end{tabular}

D, Peroxisome proliferator-activated receptor $\gamma$ hub gene

\begin{tabular}{lc}
\hline Condition & Reference counting $^{\mathrm{a}}$ \\
\hline Diabetes mellitus & 334 \\
Diabetic angiopathies & 11 \\
Diabetic cardiomyopathies & 26 \\
Diabetic nephropathies & 116 \\
Diabetic neuropathies & 49 \\
Diabetic retinopathy & 14 \\
Microvascular complications of diabetes & 0 \\
\hline
\end{tabular}

E, Superoxide dismutase 2, mitochondrial hub gene

\section{Condition}

Reference counting $^{\mathrm{a}}$

Diabetes mellitus

331
Table I. Continued.

\begin{tabular}{lc}
\hline Condition & Reference counting $^{\mathrm{a}}$ \\
\hline Diabetic angiopathies & 10 \\
Diabetic cardiomyopathies & 25 \\
Diabetic nephropathies & 121 \\
Diabetic neuropathies & 52 \\
Diabetic retinopathy & 15 \\
Microvascular complications of diabetes & 1 \\
\hline
\end{tabular}

F, Tumor necrosis factor hub gene

\begin{tabular}{lc}
\hline Condition & Reference counting $^{\mathrm{a}}$ \\
\hline Diabetes mellitus & 358 \\
Diabetic angiopathies & 12 \\
Diabetic cardiomyopathies & 28 \\
Diabetic nephropathies & 135 \\
Diabetic neuropathies & 56 \\
Diabetic retinopathy & 26 \\
Microvascular complications of diabetes & 0
\end{tabular}

$\mathrm{G}$, Transforming growth factor $\beta 1$ hub gene

Condition Reference counting ${ }^{\mathrm{a}}$

Diabetes mellitus $\quad 339$

Diabetic angiopathies $\quad 12$

Diabetic cardiomyopathies 27

Diabetic nephropathies $\quad 132$

Diabetic neuropathies $\quad 51$

Diabetic retinopathy 25

Microvascular complications of diabetes $\quad 0$

$\mathrm{H}$, Angiotensin I converting enzyme hub gene

Condition Reference counting ${ }^{\mathrm{a}}$

$\begin{array}{lr}\text { Diabetes mellitus } & 320 \\ \text { Diabetic angiopathies } & 10 \\ \text { Diabetic cardiomyopathies } & 24 \\ \text { Diabetic nephropathies } & 116 \\ \text { Diabetic neuropathies } & 47 \\ \text { Diabetic retinopathy } & 14 \\ \text { Microvascular complications of diabetes } & 1\end{array}$

${ }^{a}$ Reference counting represents the number of references regarding the association between the gene and the disease in the comparative toxicogenomics database.

processes, responses to external stimuli, blood circulation, cell death and apoptosis, and responses to oxygen levels. A clustering strategy was used to simplify these biological processes into 22 categories that were largely associated with metabo- 
A

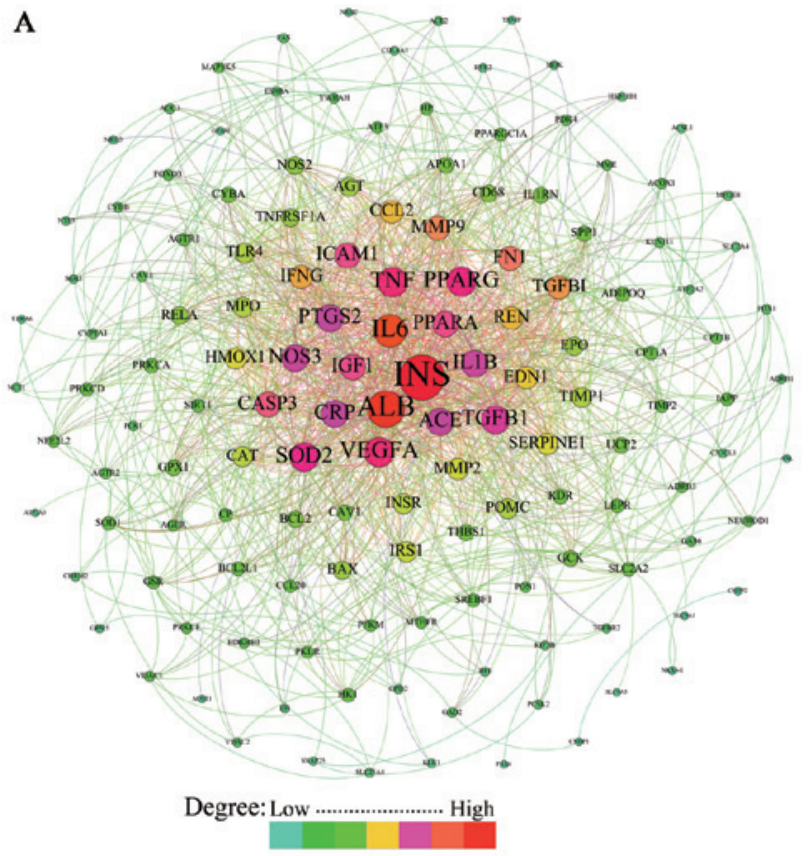

B

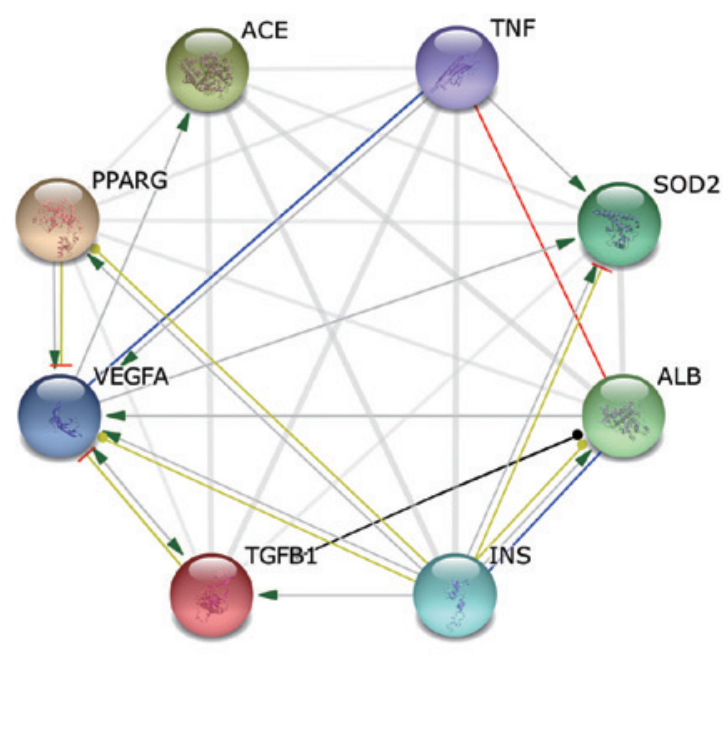

Figure 3. Hub nodes of biomarker-target networks. (A) Nodes in the biomarker-target networks were sorted according to node degree. As the node degree increases, the size and color of the nodes gradually change from small to large and green to red, respectively. (B) As an example of interactive hub nodes, eight hubs with high ranks were mapped to form a micro-interaction network based on the Search Tool for Recurring Instances of Neighboring Genes database.

A

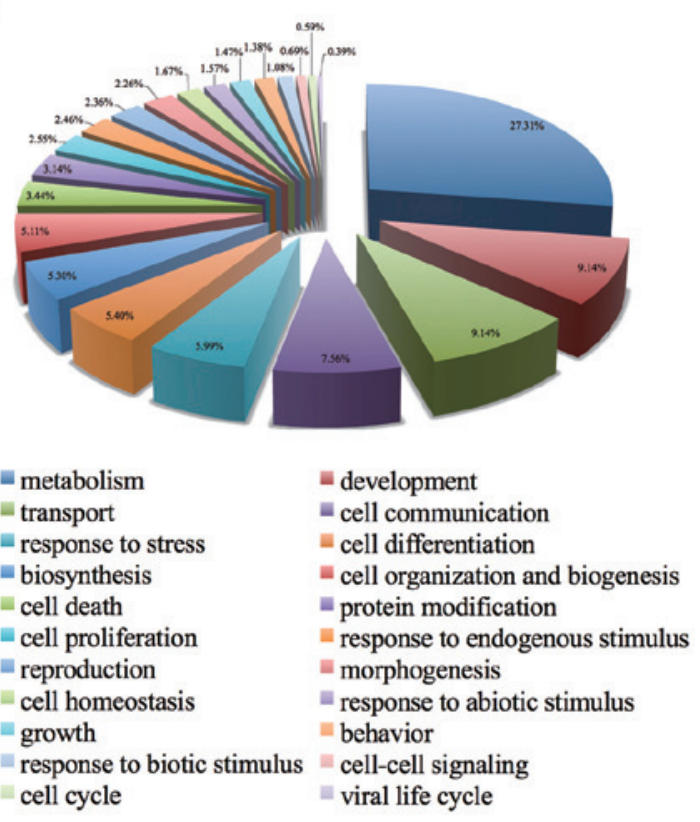

B TYPE II DAABETES NELLITUS

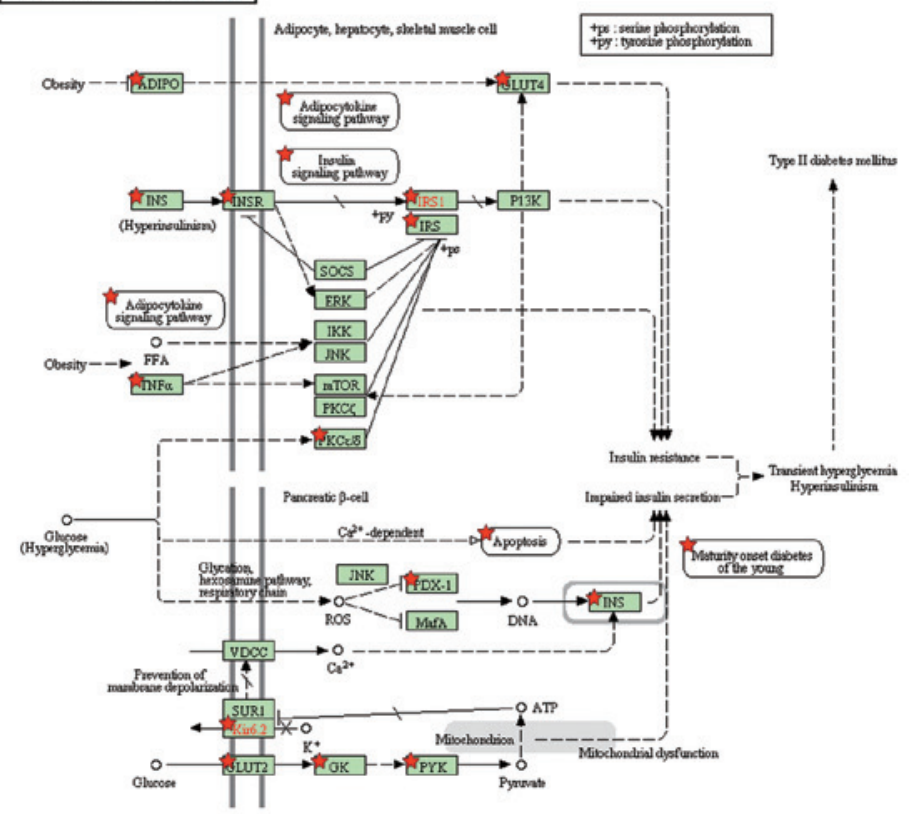

Figure 4. Enrichment analyses of biomarker-target networks. (A) A total of 142 molecular nodes were annotated by functional enrichment analysis. The results classified 1,087 biological processes into 22 categories by clustering and ratio formation. (B) The type 2 diabetes mellitus pathway from the KEGG database (www.kegg.jp/kegg/pathway.html) is presented as a statistically significant example. Molecules and pathways associated with this pathway that were identified by the present study are marked with a red star.

lism, development, cell communication, cell death and stress responses (Fig. 4A). A total of fifteen significant KEGG pathways were detected in the molecular target-biomarker network (Table III). The type 2 diabetes mellitus pathway from the KEGG database (www.kegg.jp/kegg/pathway.html) was used as an example based on its significant P-value (Fig. 4B). The molecules and pathways associated with type 2 diabetes mellitus that were identified by the present study are indicated by red stars. A total of two aspects of this pathway and the crosstalk between other pathways were highlighted (red stars, Fig. 4B) to reflect the collective dynamics of molecules and pathways. Among the fifteen pathways, the appearance of overlapping molecules is frequent, including INS and TNF, which are shared molecules of multiple pathways. 
Table II. Top 30 significant statistical biological processes.

Gene ontology

\begin{tabular}{ll}
\hline Biological process & P-value \\
\hline Homeostatic process & $1.3 \mathrm{E}-26$ \\
Chemical homeostasis & $1.1 \mathrm{E}-23$ \\
Response to organic substance & $6.0 \mathrm{E}-20$ \\
Blood circulation & $1.2 \mathrm{E}-19$ \\
Circulatory system process & $1.2 \mathrm{E}-19$ \\
Regulation of response to external & $7.0 \mathrm{E}-19$ \\
stimulus & \\
Regulation of cell proliferation & $9.7 \mathrm{E}-19$ \\
Regulation of blood pressure & $4.1 \mathrm{E}-18$ \\
Response to extracellular stimulus & $4.7 \mathrm{E}-18$ \\
Response to nutrient levels & $4.1 \mathrm{E}-18$ \\
Negative regulation of multicellular & $1.4 \mathrm{E}-17$ \\
organismal process & \\
Response to steroid hormone stimulus & $4.9 \mathrm{E}-16$ \\
Response to hormone stimulus & $1.1 \mathrm{E}-15$ \\
Response to wounding & $5.1 \mathrm{E}-15$ \\
Glucose homeostasis & $6.9 \mathrm{E}-15$ \\
Carbohydrate homeostasis & $6.9 \mathrm{E}-15$ \\
Regulation of protein amino acid & $9.3 \mathrm{E}-15$ \\
phosphorylation & \\
Regulation of protein kinase cascade & $1.8 \mathrm{E}-14$ \\
Response to endogenous stimulus & $1.7 \mathrm{E}-14$ \\
Regulation of cell death & $1.6 \mathrm{E}-14$ \\
Regulation of apoptosis & $9.3 \mathrm{E}-14$ \\
Regulation of system process & $1.2 \mathrm{E}-13$ \\
Response to hypoxia & $1.1 \mathrm{E}-13$ \\
Positive regulation of locomotion & $2.1 \mathrm{E}-13$ \\
Response to oxygen levels & $3.4 \mathrm{E}-13$ \\
organismal process & $4.9 \mathrm{E}-13$ \\
\hline & $5.9 \mathrm{E}-13$ \\
Regulation of locomotion & $1.7 \mathrm{E}-12$ \\
\hline
\end{tabular}

The threshold of EASE score, a modified fisher exact P-value (maximum probability) ranging from 0 to 1 , was used for gene-enrichment analysis. A fisher exact P-value of 0 represents perfect enrichment. In the database for annotation, visualization and integrated discovery system, fisher exact test was performed to measure the gene-enrichment in annotation terms. The smaller the value, the more enriched the gene.

\section{Discussion}

Additional systematic evidence is required to describe diabetes and its complications in a coordinated manner. A total of two notable phenomena were observed: i) Biomarkers tended to cooperate, whereas therapeutic targets were independent; and ii) nodes between diabetes and its complications tended
Table III. Significant pathways detected in the molecular target-biomarker network.

\begin{tabular}{ll}
\hline Pathway & P-value \\
\hline Type 2 diabetes mellitus & $1.1 \mathrm{E}-9$ \\
Adipocytokine signaling pathway & $7.2 \mathrm{E}-8$ \\
Maturity-onset diabetes of the young & $1.3 \mathrm{E}-6$ \\
Renin-angiotensin system & $2.9 \mathrm{E}-5$ \\
Amyotrophic lateral sclerosis (ALS) & $3.9 \mathrm{E}-5$ \\
Calcium signaling pathway & $4.7 \mathrm{E}-4$ \\
PPAR signaling pathway & $1.9 \mathrm{E}-3$ \\
Pathways in cancer & $3.4 \mathrm{E}-3$ \\
Aldosterone-regulated sodium reabsorption & $3.1 \mathrm{E}-3$ \\
Apoptosis & $6.7 \mathrm{E}-3$ \\
Cytokine-cytokine receptor interaction & $1.7 \mathrm{E}-2$ \\
Type 1 diabetes mellitus & $2.0 \mathrm{E}-2$ \\
Insulin signaling pathway & $2.4 \mathrm{E}-2$ \\
Huntington's disease & $4.4 \mathrm{E}-2$ \\
Neurotrophin signaling pathway & $4.6 \mathrm{E}-2$
\end{tabular}

The threshold of EASE score, a modified fisher exact P-value (maximum probability) ranging from 0 to 1 , was used for gene-enrichment analysis. A fisher exact P-value of 0 represents perfect enrichment. In the database for annotation, visualization and integrated discovery system, fisher exact test was performed to measure the gene-enrichment in annotation terms. The smaller the value, the more enriched the gene.

to reveal molecular communication, whereas there appeared to be no contact between complications. Furthermore, the colorful subnetwork reflected dense internal communication trends and extensive crosstalk-like structures between diabetes and its complications. These horizontal and vertical molecular regulatory modes suggest common triggers of pathological evolution, and thus a requirement for multi-target intervention.

The results of the present study are consistent with previous studies of network models of complex disease interventions and key regulatory pathways for type 2 diabetes-associated risk/complications based on gene co-expression networks $(20,21)$. These studies indicated that targeting a single node is unlikely to affect a disease network (22) and multiple proteins must be regulated to affect disease phenotypes. Network intervention emphasizes the importance of communication infrastructures, and the linkages between biomarkers and targets may involve direct or indirect connections. The majority of biotherapeutic drugs are hypothesized to act on specific targets, which may serve as biomarkers for target engagement and validation $(23,24)$. A systems biology approach to investigating disease-associated biology is revolutionizing our understanding of the cellular pathways and gene networks underlying the onset of disease and the mechanisms underlying pharmacological treatments that ameliorate disease phenotypes $(25,26)$, thereby facilitating drug discovery and development through the identification of novel drug targets and biomarkers of disease progression.

In the present study, various topological properties of a biomarker-target network were revealed, which may provide future targets for potential systematic interventions. Hub 
nodes are the backbone of the network topological architecture and may improve our understanding of molecular integration. Similar results have been reported for transcriptional networks (27), protein-protein interaction networks (28), gene co-expression networks (29) and regulatory networks (30). The robustness of a hub gene network formed by various associated diseases is indicated by its highly connected components. Strong correlations between the expression levels of hub genes and indicators of disease severity suggest that this hub network may partially reflect disease progression (31). Consequently, it is important to investigate not only the network structure based on hub nodes but also the parallel role of anti-structure.

The collective behavior and compound effects of the hubs are hypothesized to be generated based on three distinct aspects. Separate molecules may have a common downstream target. For example, the early stages of endothelial dysfunction in diabetes may be associated with the increased expression of INS, VEGFs, TGF $\beta 1$ and TNF, which in addition are suspected to be critical in the pathogenesis of diabetic angiopathy (32). Research suggests that these four pro-angiogenic factors may be involved in the pathology of diabetic retinopathy, heart disease and atherosclerosis-based microangiopathy (33). The present study speculates that centralized regulation is the primary pattern for consistent targeted expression of integrated regulation.

In addition, interactive cooperation may occur between molecules. In contrast to consistent targeting, intermolecular cooperation emphasizes directed execution, as indicated by evidence of extensive information transmission in the field of systems biology (31). For example, SOD2 is a member of the iron/manganese superoxide dismutase family, which likely contributes to the upregulation of microRNAs responsible for regulating VEGF. These data led to an improved understanding of the role of epigenetics in diabetic retinopathy (34). A similar interaction occurs between TGF $\beta$ and VEGF to enhance the production of VEGF by retinal cells following injuries complicated by diabetes and hypoxia in the retina (32). Thus, this molecular interaction depends on cooperation as the basic paradigm of integrated regulation.

Furthermore, single molecules may perform multiple roles. Genetic pleiotropy occurs when one gene affects the expression of multiple characteristics. For example, elevated VEGF levels and low levels of VEGF activity act as pathological stimuli in ocular neovascularization and peripheral neuropathy, respectively (35). Anti-VEGF approaches have been applied in clinical settings due to the induction of abnormal angiogenesis and enhanced retinal capillary permeability by the overproduction of VEGF (36). However, high VEGF expression exhibits beneficial effects on diabetic nephropathy via unknown underlying mechanisms (37). This evidence demonstrates that an objective analytical framework of multi-disease integrated regulation that indicates a horizontal and vertical combination of various core molecules or overall members may provide a robust basis for common diagnosis or integrated therapies for diabetes and its complications.

Abnormal INS signaling driven by obesity and hyperglycemia may be responsible for INS resistance and impaired INS secretion, leading to the spatio-temporal initiation of continuous and devastating metabolic processes. This conditional collapse involves a complex signaling network, including INS resistance, $\beta$-cell dysfunction, impaired glucose tolerance and mitochondrial dysfunction activated by the INS receptor, which regulates intermediary metabolism and ultimately leads to diabetic disease states, including types 1 and 2 diabetes mellitus and maturity-onset diabetes in youth $(38,39)$. Adipocytokines and glycemia partially account for the association between adiposity and the risk of type 2 diabetes (40) and are important in the pathogenesis of INS resistance and associated metabolic complications, including dyslipidemia, hypertension and premature heart disease (41). Atherosclerosis is considered to be the predominant cause of morbidity and mortality in patients with diabetes mellitus (42). Epidemiological evidence suggests that the risk of various types of cancer is increased in patients with diabetes $(43,44)$ and that obesity, hyperglycemia and enhanced oxidative stress may contribute to this increased risk. Furthermore, cytokine-cytokine receptor-associated pathways may be potential biological links between diabetes and cancer risk. Hyperglycemia may influence multiple signaling pathways, including INS and INS-like growth factor receptor signaling, to stimulate multiple cancer phenotypes, including proliferation, protection from apoptotic stimuli, invasion and metastasis, potentially enhancing the promotion and progression of numerous types of cancer cells $(44,45)$. Programmed cell death in diabetes and its complications occurs in pancreatic $\beta$-cells and neural and vascular cells, resulting in retinopathy, neuropathy, nephropathy and cardiovascular disease via calcium signaling, cytokine signaling, hyperglycemia and mitochondrial damage (46-49). Furthermore, neurotrophins have been assessed as candidate therapeutic agents for the treatment of neural complications of diabetes $(50,51)$; these complications may result from the promotion of neovascularization and microvascular neuroprotection. Taken together, these data indicate the extreme complexity of molecular integrated regulation between diabetes and its complications. Based on convergent biofunctions, the crosstalk between multiple pathways may be exploited to reverse complex pathological cascades.

Integrated molecular pathological analysis may provide a novel strategy with which to improve our understanding of associated diseases. In the present study, large-scale, interlocking molecular cooperation between diabetes and its complications was demonstrated. Systems analysis is based on the characteristics of molecules, including common downstream targets, interactive cooperation between molecules and multiple roles of single molecules. The shared underlying mechanisms involve endothelial dysfunction, inflammatory factor mediation, neovascularization, neuronal oxidative injury, cell death and apoptosis, lipometabolism and mitochondrial damage. Integrated regulated analysis strategies may be used to reveal general trends, to dissect underlying pathological mechanisms and for drug screening $(26,52)$. The identification of network-coupled biomarkers and therapeutic targets may facilitate molecular diagnoses and treatments. In conclusion, this may be the beginning of a renewed fight against diabetes and its complicationfuture studies may focus on blocking pathological cascades through the integrated regulation of key molecules and pathway crosstalk.

\section{References}

1. Shaw JE, Sicree RA and Zimmet PZ: Global estimates of the prevalence of diabetes for 2010 and 2030. Diabetes Res Clin Pract 87: 4-14, 2010. 
2. Chen L, Magliano DJ and Zimmet PZ: The worldwide epidemiology of type 2 diabetes mellitus-present and future perspectives. Nat Rev Endocrinol 8: 228-236, 2012.

3. van Dieren S: Management of type 2 diabetes mellitus and prediction of cardiovascular complications. Thesis, Utrecht University. ISBN: 978-94-6203-340-5, 2013.

4. Tabit CE, Chung WB, Hamburg NM and Vita JA: Endothelia dysfunction in diabetes mellitus: Molecular mechanisms and clinical implications. Rev Endocr Metab Disord 11: 61-74, 2010.

5. Giacco F and Brownlee M: Oxidative stress and diabetic complications. Circ Res 107: 1058-1070, 2010

6. Donath MY and Shoelson SE: Type 2 diabetes as an inflammatory disease. Nat Rev Immunol 11: 98-107, 2011.

7. Pedicino D, Liuzzo G, Trotta F, Giglio AF, Giubilato S, Martini F, Zaccardi F, Scavone G, Previtero M, Massaro G, et al: Adaptive immunity, inflammation, and cardiovascular complications in type 1 and type 2 diabetes mellitus. J Diabetes Res 2013: 184258, 2013

8. Sivitz WI and Yorek MA: Mitochondrial dysfunction in diabetes: From molecular mechanisms to functional significance and therapeutic opportunities. Antioxid Redox Signal 12: 537-577, 2010.

9. Zhong Q and Kowluru RA: Epigenetic changes in mitochondrial superoxide dismutase in the retina and the development of diabetic retinopathy. Diabetes 60: 1304-1313, 2011.

10. van Dieren S, Beulens JW, van der Schouw YT, Grobbee DE and Neal B: The global burden of diabetes and its complications: An emerging pandemic. Eur J Cardiovasc Prev Rehabil 17 (Suppl 1): S3-S8, 2010.

11. Sladek R, Rocheleau G, Rung J, Dina C, Shen L, Serre D, Boutin P, Vincent D, Belisle A, Hadjadj S, et al: A genome-wide association study identifies novel risk loci for type 2 diabetes. Nature 445: 881-885, 2007.

12. Zeggini E, Weedon MN, Lindgren CM, Frayling TM, Elliott KS Lango H, Timpson NJ, Perry JR, Rayner NW, Freathy RM, et al: Replication of genome-wide association signals in UK samples reveals risk loci for type 2 diabetes. Science 316: 1336-1341, 2007.

13. Barrett JC, Clayton DG, Concannon P, Akolkar B, Cooper JD, Erlich HA, Julier C, Morahan G, Nerup J, Nierras C, et al: Genome-wide association study and meta-analysis find that over 40 loci affect risk of type 1 diabetes. Nat Genet 41: 703-707, 2009.

14. Voight BF, Scott LJ, Steinthorsdottir V, Morris AP, Dina C, Welch RP, Zeggini E, Huth C, Aulchenko YS, Thorleifsson G, et al Twelve type 2 diabetes susceptibility loci identified through large-scale association analysis. Nat Genet 42: 579-589, 2010.

15. Pezzolesi MG, Poznik GD, Mychaleckyj JC, Paterson AD, Barati MT, Klein JB, Ng DP, Placha G, Canani LH, Bochenski J, et al: Genome-wide association scan for diabetic nephropathy susceptibility genes in type 1 diabetes. Diabetes 58 : 1403-1410, 2009.

16. Pirola L, Balcerczyk A, Okabe J and El-Osta A: Epigenetic phenomena linked to diabetic complications. Nat Rev Endocrinol 6: 665-675, 2010

17. Reddy MA and Natarajan R: Epigenetic mechanisms in diabetic vascular complications. Cardiovasc Res 90: 421-429, 2011.

18. von Mering C, Huynen M, Jaeggi D, Schmidt S, Bork P and Snel B: STRING: A database of predicted functional associations between proteins. Nucleic Acids Res 31: 258-261, 2003.

19. Watts DJ and Strogatz SH: Collective dynamics of 'small-world' networks. Nature 393: 440-442, 1998

20. Rende D, Baysal N and Kirdar B: Complex disease interventions from a network model for type 2 diabetes. PloS One 8: e65854, 2013

21. Sengupta U,Ukil S, Dimitrova N and Agrawal S: Expression-based network biology identifies alteration in key regulatory pathways of type 2 diabetes and associated risk/complications. PloS One 4 e8100,2009.

22. Hopkins AL: Network pharmacology: The next paradigm in drug discovery. Nat Chem Biol 4: 682-690, 2008.

23. Lee JW and Salimi-Moosavi H: Bioanalysis of target biomarker and PK/PD relevancy during the development of biotherapeutics. Bioanalysis 4: 2513-2523, 2012.

24. Liu Z, Cort L, Eberwine R, Herrmann T, Leif JH, Greiner DL, Yahalom B, Blankenhorn EP and Mordes JP: Prevention of type 1 diabetes in the rat with an allele-specific anti-T-cell receptor antibody: V $\beta 13$ as a therapeutic target and biomarker. Diabetes 61: 1160-1168, 2012.

25. Wang IM, Stone DJ, Nickle D, Loboda A, Puig O and Roberts C: Systems biology approach for new target and biomarker identification. Curr Top Microbiol Immunol 363: 169-199, 2013.

26. Wang $Z$ and Wang $Y$ : Navigating personalized medicine dependent on modular flexibility. Trends Mol Med 19: 393-395, 2013.
27. Santana-Codina N, Carretero R, Sanz-Pamplona R, Cabrera T, Guney E, Oliva B, Clezardin P, Olarte OE, Loza-Alvarez P, Méndez-Lucas A, et al: A transcriptome-proteome integrated network identifies endoplasmic reticulum thiol oxidoreductase (ERp57) as a hub that mediates bone metastasis. Mol Cell Proteomics 12: 2111-2125, 2013.

28. Luo T, Wu S, Shen X and Li L: Network cluster analysis of protein-protein interaction network identified biomarker for early onset colorectal cancer. Mol Biol Rep 40: 6561-6568, 2013.

29. Zhou Y, Xu J, Liu Y, Li J, Chang C and Xu C: Rat hepatocytes weighted gene co-expression network analysis identifies specific modules and hub genes related to liver regeneration after partial hepatectomy. PloS One 9: e94868, 2014.

30. Mahdi R, Madduri AS, Wang G, Strulovici-Barel Y, Salit J, Hackett NR, Crystal RG and Mezey JG: Empirical Bayes conditional independence graphs for regulatory network recovery. Bioinformatics 28: 2029-2036, 2012.

31. Liang D, Han G, Feng X, Sun J, Duan Y and Lei H: Concerted perturbation observed in a hub network in Alzheimer's disease. PloS One 7: e40498, 2012.

32. Stehouwer CD, Lambert J, Donker AJ and van Hinsbergh VW: Endothelial dysfunction and pathogenesis of diabetic angiopathy. Cardiovasc Res 34: 55-68, 1997.

33. Xu L, Kanasaki K, Kitada M and Koya D: Diabetic angiopathy and angiogenic defects. Fibrogenesis Tissue Repair 5: 13, 2012.

34. Kowluru RA, Santos JM and Mishra M: Epigenetic modifications and diabetic retinopathy. Biomed Res Int 2013: 635284, 2013

35. Wirostko B, Wong TY and Simó R: Vascular endothelial growth factor and diabetic complications. Prog Retin Eye Res 27: 608-621, 2008.

36. Alghadyan AA: Diabetic retinopathy-An update. Saudi J Ophthalmol 25: 99-111, 2011.

37. Sivaskandarajah GA, Jeansson M, Maezawa Y, Eremina V, Baelde HJ and Quaggin SE: Vegfa protects the glomerular microvasculature in diabetes. Diabetes 61: 2958-2966, 2012.

38. Saltiel AR and Kahn CR: Insulin signalling and the regulation of glucose and lipid metabolism. Nature 414: 799-806, 2001.

39. Rains JL and Jain SK: Oxidative stress, insulin signaling, and diabetes. Free Radic Biol Med 50: 567-575, 2011.

40. Kanaya AM, Wassel Fyr C, Vittinghoff E, Harris TB, Park SW, Goodpaster BH, Tylavsky F and Cummings SR: Adipocytokines and incident diabetes mellitus in older adults: The independent effect of plasminogen activator inhibitor 1. Arch Intern Med 166: 350-356, 2006

41. Rasouli N and Kern PA: Adipocytokines and the metabolic complications of obesity. J Clin Endocrinol Metab 93 (11 Suppl 1): S64-S73, 2008.

42. Beckman JA, Creager MA and Libby P: Diabetes and atherosclerosis: Epidemiology, pathophysiology, and management. JAMA 287: 2570-2581, 2002

43. Vigneri P,Frasca F, Sciacca L, Pandini G and Vigneri R: Diabetes and cancer. Endocr Relat Cancer 16: 1103-1123, 2009.

44. Giovannucci E, Harlan DM, Archer MC, Bergenstal RM, Gapstur SM, Habel LA, Pollak M, Regensteiner JG and Yee D: Diabetes and cancer: A consensus report. CA Cancer J Clin 60 : 207-221, 2010.

45. Wolf I, Sadetzki S, Catane R, Karasik A and Kaufman B: Diabetes mellitus and breast cancer. Lancet Oncol 6: 103-111, 2005.

46. Mandrup-Poulsen T: Apoptotic signal transduction pathways in diabetes. Biochem Pharmacol 66: 1433-1440, 2003.

47. Allen DA, Yaqoob MM and Harwood SM: Mechanisms of high glucose-induced apoptosis and its relationship to diabetic complications. J Nutr Biochem 16: 705-713, 2005.

48. Barber AJ, Gardner TW and Abcouwer SF: The significance of vascular and neural apoptosis to the pathology of diabetic retinopathy. Invest Ophthalmol Vis Sci 52: 1156-1163, 2011.

49. Bhatt MP, Lim YC, Kim YM and Ha KS: C-peptide activates AMPK $\alpha$ and prevents ROS-mediated mitochondrial fission and endothelial apoptosis in diabetes. Diabetes 62: 3851-3862, 2013.

50. Vincent AM, Callaghan BC, Smith AL and Feldman EL: Diabetic neuropathy: Cellular mechanisms as therapeutic targets. Nat Rev Neurol 7: 573-583, 2011.

51. Navaratna D, Guo SZ, Hayakawa K, Wang X, Gerhardinger C and Lo EH: Decreased cerebrovascular brain-derived neurotrophic factor-mediated neuroprotection in the diabetic brain. Diabetes 60: 1789-1796, 2011.

52. Zhang YY, Li HX, Chen YY, Fang H, Yu YN, Liu J, Jing ZW, Wang Z and Wang YY: Convergent and divergent pathways decoding hierarchical additive mechanisms in treating cerebral ischemia-reperfusion injury. CNS Neurosci Ther 20: 253-263, 2014. 